Competencias docentes, una innovación en ambientes virtuales de aprendizaje en
Teaching skills, an innovation in

\section{virtual learning environments}

in higher education

\title{
educación superior
}

\author{
Carlos Arturo Vega Lebrún* | Mariano Sánchez Cuevas** | Genoveva Rosano Ortega*** | Silvia Elena Amador Pérez****
}

Recepción del artículo: 29/03/2021 | Aceptación para publicación: 22/06/2021 | Publicación: 30/09/2021

\section{RESUMEN}

El cierre de emergencia de las instituciones educativas ante la contingencia sanitaria por la covid-19 ha apremiado el replanteamiento de los modelos de competencias docentes para adaptarse a los ambientes virtuales de aprendizaje (AVA). El objetivo de este estudio fue diseñar y validar un modelo de competencias docentes en AVA, con el soporte de laboratorios virtuales, a fin de asegurar la calidad de la educación superior en este contexto. Se empleó una metodología cuantitativa y correlacional en un estudio de caso. La investigación se dividió en dos momentos metodológicos: el diseño del modelo y su validación. Los instrumentos utilizados fueron una matriz de análisis comparativo y una escala tipo Likert. En la etapa de validación participaron 5650 estudiantes y 41 profesores. Los resultados destacan la necesidad del desarrollo docente, principalmente en las competencias digitales y de investigación. Una debilidad del estudio es la falta de ponderación de los indicadores de calidad. El modelo propuesto representa una innovación en la definición de indicadores, en forma de competencias, para el aseguramiento de la calidad educativa en los AVA. Se concluye que el modelo propuesto es pertinente para el aseguramiento de la calidad educativa en AVA.

\section{Palabras clave}

Ambientes virtuales de aprendizaje; criterios de calidad educativa; competencias docentes; educación superior

\section{Abstract}

The emergency closure of educational institutions, in the face of the health contingency due to Covid-19, has prompted the rethinking of the models of teaching competencies to adapt to virtual learning environments (VLE). The objective of this study is to design and validate a model of teaching competencies in VLE, with the support of virtual laboratories, to ensure the quality of higher education during the covid-19 pandemic. A quantitative and correlational methodology was used in a case study. The research is divided into two methodological moments: the design of the model and its validation. The instruments used were a comparative analysis matrix and a Likert-type scale. 5650 students and 41 teachers participated in the validation cap. The results highlight the need for teacher development, mainly in digital and research skills. One weakness of the study is the lack of weighting of the quality indicators. The proposed model represents an innovation in the definition of indicators, in the form of competencies, for the assurance of educational quality in VLE. It is concluded that the proposed model is pertinent for the assurance of educational quality in $V L E$.

* Doctor en Tecnologías de Información y Análisis de Decisiones por la Universidad Popular Autónoma del Estado de Puebla, México. ORCID: https://orcid. org/0000-0002-8304-2649 |** Doctor en Pedagogía por la Universidad Popular Autónoma del Estado de Puebla, México. ORCID: https://orcid.org/0000-0003- 


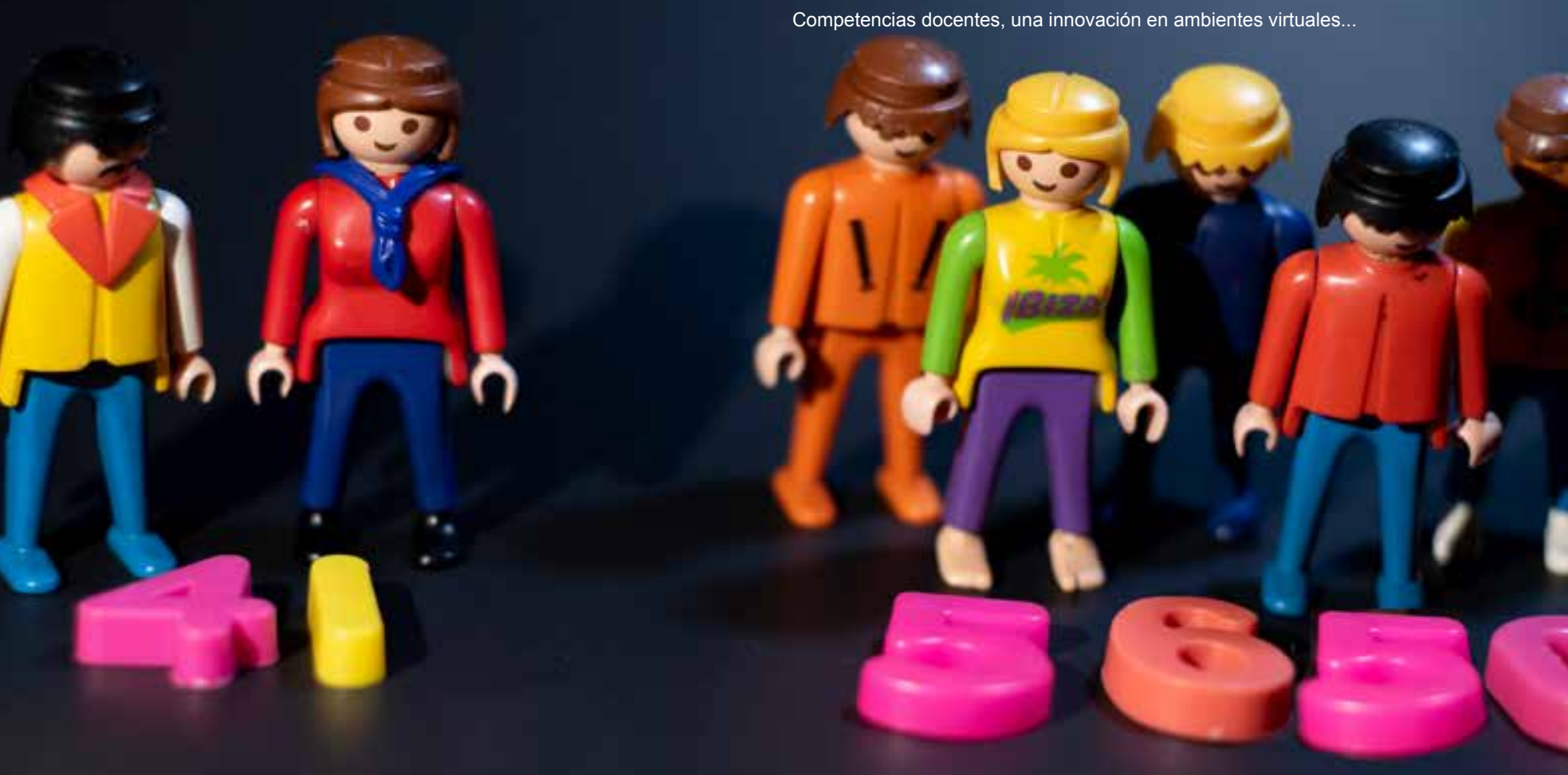

\section{INTRODUCCIÓN}

\section{La calidad de la educación en el nivel superior}

$\mathrm{L}$ as instituciones educativas se han vuelto cada vez más conscientes de la importancia de ofrecer una educación de alta calidad para satisfacer las necesidades de una diversidad de estudiantes, así como de los cambios globales y la adaptación al contexto por la pandemia de la covid-19. Esto, aunado a las diferentes modalidades educativas que existen en la educación superior (presencial, en línea o mixta), requiere que se propongan formas innovadoras de garantizar y sistematizar la calidad en estas instituciones.

Estudios recientes sobre la calidad de la educación superior (Kundu, 2017) se han enfocado principalmente en: a) modelos de calidad basados en TQM (Total Quality Management), b) calidad basada en resultados, c) calidad vista como siste- ma, d) calidad del servicio desde la perspectiva de las partes interesadas, e) brechas de calidad en la educación superior internacional, f) calidad desde la perspectiva de los estudiantes, g) calidad de los sistemas de e-learning, y h) factores críticos que determinan el éxito en la calidad educativa.

Otras revisiones (Prakash, 2018) indican que los constructos de calidad del aprendizaje, de la calidad del servicio y de la satisfacción de los estudiantes han recibido una mayor atención, seguidos de la gestión de la calidad total, el aseguramiento de la calidad y la evaluación integral en el desempeño del estudiante. A su vez, los aspectos metodológicos mayormente utilizados han sido el método empírico, las encuestas y los estudios de casos individuales. Europa lidera la investigación sobre la calidad de las instituciones de educación superior (IES), seguida de América del Norte y Asia.

Asimismo, las IES han optado por obtener acreditaciones nacionales e internacionales que 
avalen la calidad de sus programas e instituciones. En un contexto de globalización, la acreditación es una herramienta de aseguramiento de la calidad que testifica que una institución o programa haya pasado por un riguroso proceso de evaluación externa (Kumar, Shukla y Passey, 2020). Las acreditaciones de la calidad educativa han permitido implementar una cultura de rendición de cuentas, bajo la idea de que son herramientas de desarrollo y bienestar económico (Jiménez, 2019). Por este motivo, para el desarrollo del modelo propuesto se revisaron los criterios de calidad docente considerados por siete organismos acreditadores, cinco internacionales y dos nacionales, así como el análisis de los indicadores sustantivos de calidad de seis modelos educativos, tanto nacionales como internacionales (ver tabla 1).

Debido a la pandemia de la covid-19, el desafío para las IES fue adaptar o innovar sus modelos de calidad académica a la modalidad virtual, en la totalidad de su oferta educativa. Los docentes, por su parte, han atravesado dificultades como el acceso inadecuado a internet, la falta de infraestructura (brecha de acceso), el manejo de las clases virtuales y el proceso de educación a distancia en sí mismo (brecha de competencias digitales) (García-Peñalvo, Corell, Abella-García y Grande, 2020; Sari y Nayir, 2020; Barrón, 2020).

Ante el impacto de la llamada "nueva normalidad" post-pandémica, es probable que prevalezcan las formas de educación virtual o híbrida por largo tiempo (Cabero-Almenara y Llorente-Cejudo, 2020). Esto hace necesario la innovación de perfiles docentes que apuntalen las competencias para desempeñarse en los ambientes virtuales de aprendizaje. En este tenor, el modelo que aquí se presenta atiende esta necesidad emergente.

Tabla 1. Organismos acreditadores de la calidad y modelos educativos analizados

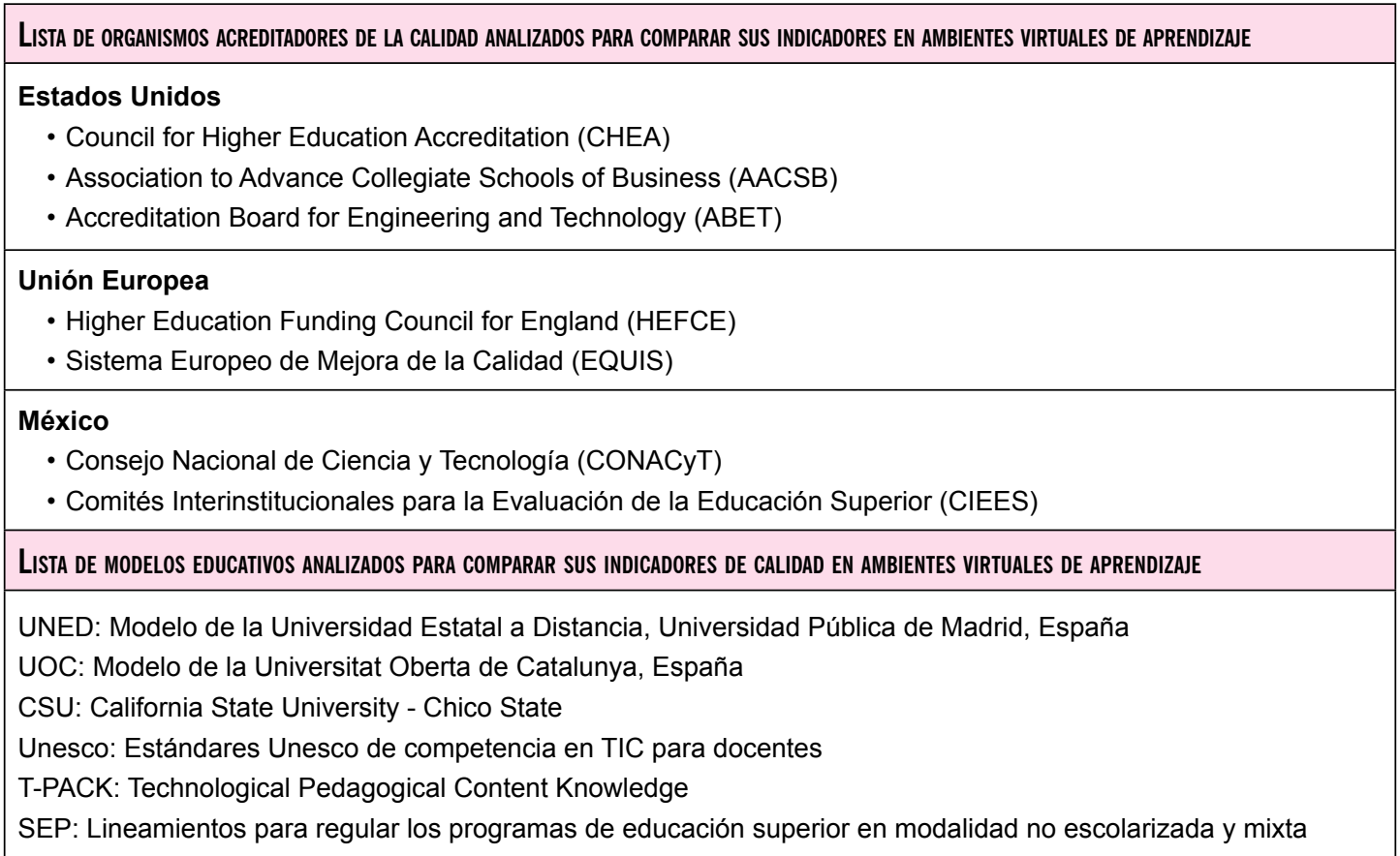

Fuente: elaboración propia. 


\section{LA EVALUACIÓN DE LA CALIDAD DESDE LA PERSPECTIVA DE SATISFACCIÓN DE LOS ESTUDIANTES}

Al considerar a los estudiantes como los beneficiarios directos de la educación superior, la calidad educativa puede ser definida como "una relación directa entre las expectativas y percepciones de las experiencias vividas" (Herrera, Souza y De Quadros, 2018, p. 73). Resulta pertinente evaluarla desde la percepción de satisfacción de los estudiantes, a través de la generación de experiencias significativas.

De este modo, para brindar servicios educativos de calidad académica, las instituciones deben entender las necesidades de sus estudiantes. Además de proveer un servicio eficiente y satisfactorio, las universidades pueden contribuir a incentivar en los estudiantes un comportamiento positivo, generar lealtad y motivación, mejorar la permanencia, los resultados de aprendizaje, el rendimiento académico, la recomendación del servicio y, por consiguiente, la sostenibilidad de la institución (Herrera et al., 2018). Adicionalmente, las percepciones positivas de los estudiantes sobre la calidad del servicio pueden conducir a una mejor imagen institucional, por lo que es vital para las IES monitorear la calidad del servicio e identificar las necesidades de su población estudiantil (Yong-Sik y Yung Kyun, 2019).
La satisfacción de los estudiantes es una dimensión importante, que pocos estudios han examinado, para verificar la calidad de la educación superior (Allam, 2018). Desde este punto de partida, algunas de las categorías que han resultado significativas para evaluar la calidad de la educación superior son: los servicios académicos, las instalaciones académicas, los servicios administrativos y de atención a los estudiantes (El Alfy y Abukari, 2020). Otras categorías en el mismo sentido son: el contenido del plan de estudios, los recursos educativos, los factores institucionales y las experiencias de enseñanza-aprendizaje (Allam, 2018).

\section{LOS AMBIENTES VIRTUALES DE APRENDIZAJE}

El cierre de aulas durante la pandemia por la covid-19 "propició una migración forzada hacia la modalidad no presencial en todos los niveles educativos" (Aguilar, 2020, p. 47). Así, las modalidades educativas no presenciales, mediadas por las tecnologías de información y comunicación (TIC), permitieron enfrentar mejor la situación. Este contexto hace pertinente consolidar lo que en la última década se ha denominado ambientes virtuales de aprendizaje (AVA), también nombrados entornos virtuales de enseñanza-aprendizaje.

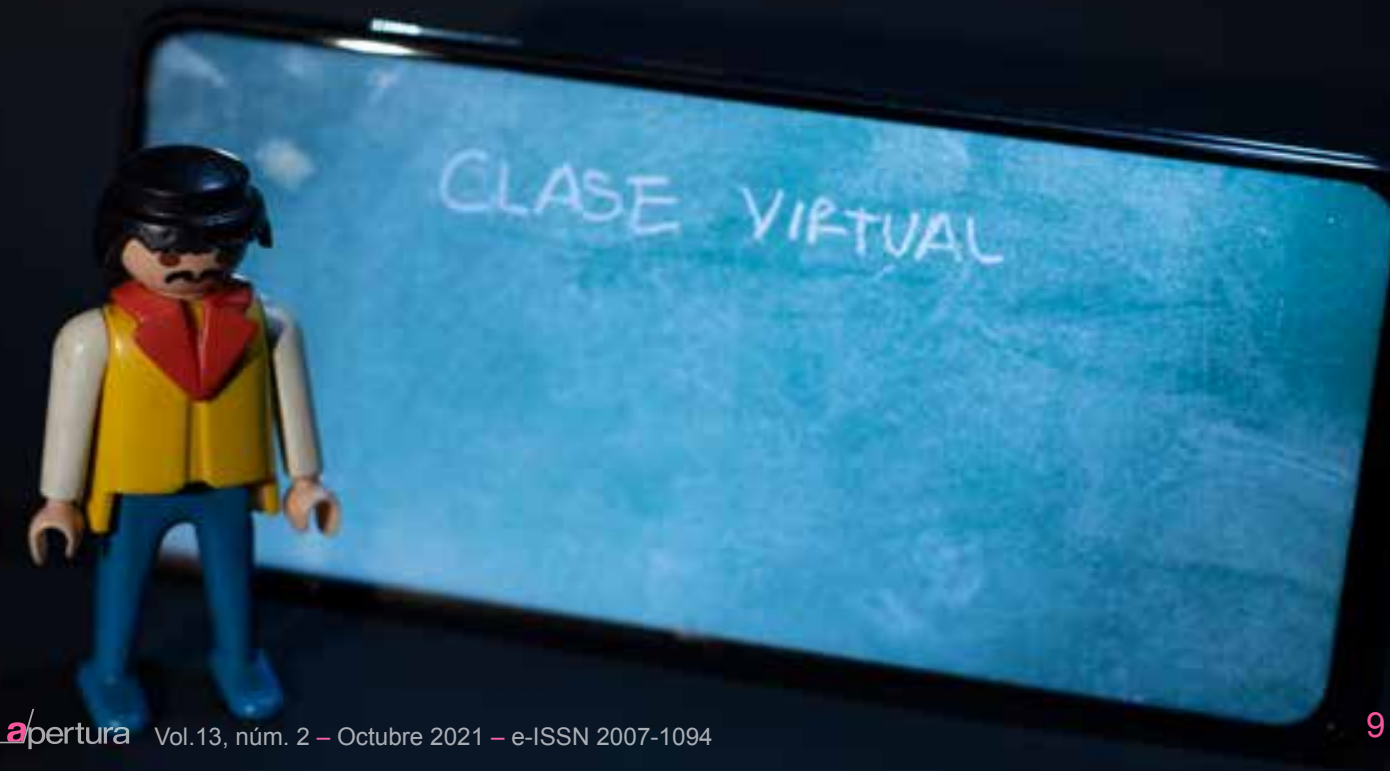


Un AVA combina los espacios físico y virtual para proveer a los usuarios una sensación de presencialidad en un ambiente virtual (Peterson-Ahmad, Pemberton y Hovey, 2018).

Inicialmente, puede entenderse que los AVA integran las TIC y lo educativo, creados con la finalidad de facilitar la docencia y promover aprendizajes (Cocunubo-Suárez, Parra-Valencia y Otálora-Luna, 2018); sin embargo, proveer a los profesores y estudiantes de plataformas electrónicas, videoconferencias síncronas y contenidos digitalizados no garantiza el aprendizaje significativo (Kundu, 2017; García-Peñalvo et al., 2020). Además, es necesario el compromiso y soporte institucional para establecer claramente las dimensiones de calidad en esta modalidad educativa. Esto permite acompañar, evaluar y motivar a los docentes -en contextos como el actual, que ha sido impactado por la pandemia- cuando muchos de ellos no se sienten preparados para esta transición hacia la virtualidad (Scherer, Howard, Tondeur y Siddiq, 2020).

Lograr la calidad en los AVA depende fundamentalmente de la acción del docente como diseñador de andamiajes educativos que coloquen al estudiante en un rol central y activo. Esto puede lograrse mediante metodologías activas, como el aprendizaje basado en problemas y contextos (Phungsuk, Viriyavejakul y Ratanaolarn, 2017), el aprendizaje basado en retos (Sánchez, 2020) y el aprendizaje colaborativo (García-Chitiva y Suárez-Guerrero, 2019).

Los AVA abarcan una amplia gama de recursos que incluyen las páginas web, los sistemas de gestión de aprendizaje y los entornos de aprendizaje tridimensionales (Khlaisang y Songkram, 2019). Entre estos últimos destacan los laboratorios virtuales como una innovación para enfrentar el hecho de que, a causa de la pandemia, los estudiantes no pueden acceder a los laboratorios en forma presencial dentro de las universidades (Luna, Costales y Cordovez, 2021).

Un laboratorio virtual puede definirse como un ambiente interactivo para la creación y conducción de experimentos simulados. Algunas ventajas de implementarlos son: a) el estudiante puede enfrentar situaciones que podrían ser inseguras en otros contextos, b) puede experimentar sin riesgos, y c) el tiempo y espacio para las prácticas es más flexible que en los ambientes físicos (SalmerónManzano y Manzano-Agugliaro, 2018). Asimismo, se ha demostrado que los laboratorios virtuales son óptimos para lograr la transferencia de conocimiento en diversas disciplinas (Bonilla, Villamil y Montes, 2019; González y Victoria, 2019; Nechypurenko, Selivanova y Chernova, 2019).

\section{COMPETENCIAS DOCENTES Y DIGITALES EN EDUCACIÓN SUPERIOR}

Junto con la situación de pandemia por la covid-19, existen otras fuerzas que orillan a modificar el rol del docente, entre las que se puede mencionar la cuarta revolución industrial, las pedagogías innovadoras, la explosión de información por el uso de internet, la inteligencia artificial y el desplazamiento hacia modalidades de educación masiva abierta (Ally, 2019). No obstante, coexisten fuerzas que frenan la innovación necesaria del rol docente; por ejemplo, la mayoría de los profesores carece de formación especializada en educación superior, los estudiantes están mayormente familiarizados con las clases tradicionales, los currículos y los exámenes están diseñados desde la enseñanza tradicional (Zempoalteca, Barragán, González y Guzmán, 2016). A pesar de esto, es necesario que el perfil docente se aparte de los modelos educativos tradicionales y transite hacia roles innovadores. Las diversas competencias de este perfil deben incluir el uso educativo de las TIC, la aplicación de innovaciones pedagógicas y la capacidad de investigación, entre otras.

Se entiende por competencias a los procesos complejos de desempeño con idoneidad en determinados contextos, a partir de la integración de diferentes saberes (saber ser, saber hacer, saber conocer y saber convivir) para realizar actividades 
y resolver problemas con sentido de reto, motivación, flexibilidad, creatividad, comprensión y emprendimiento, dentro de una perspectiva de procesamiento metacognitivo, mejoramiento continuo y compromiso ético. Esto, con la meta de contribuir al desarrollo personal, la construcción y afianzamiento del tejido social, la búsqueda continua del desarrollo económico-empresarial sostenible, así como el cuidado y la protección del ambiente (Tobón, 2008). El enfoque por competencias facilita la evaluación de la calidad de la formación (o de la capacitación) y la calidad del desempeño, además de que puede orientar la certificación de competencias profesionales.

Existen diversos estudios y propuestas acerca de cuáles son las competencias genéricas docentes requeridas en el nivel de educación superior, que incluyen el diseño instruccional, las estrategias didácticas, la evaluación educativa, la comunicación, el liderazgo y la investigación como las más frecuentes (Villarroel y Bruna, 2017; MacíasCatagua, 2018; Clavijo, 2018). Las competencias para el uso de las tecnologías digitales se han convertido en un requisito para los docentes de

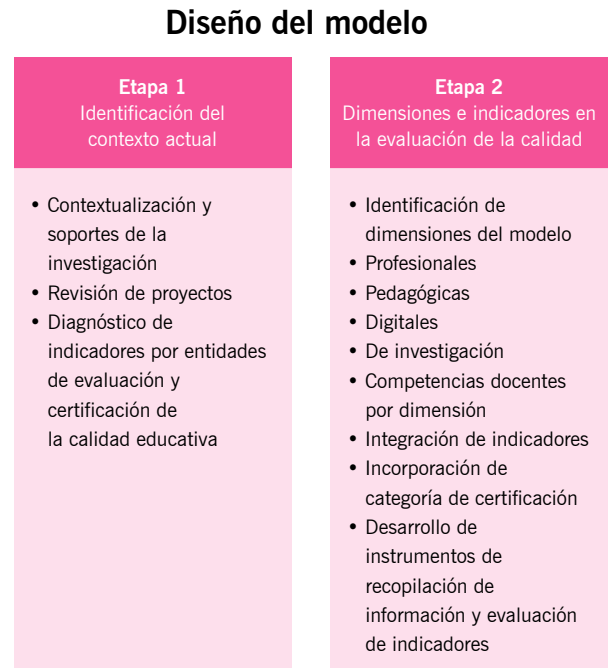

educación superior a nivel global. Al respecto, Albrahim (2020) concluyó que las competencias que se requieren para la enseñanza efectiva en ambientes de aprendizaje en línea se pueden clasificar en seis categorías: a) habilidades pedagógicas, b) de manejo de contenido, c) para el diseño, d) tecnológicas, e) administrativas y f) institucionales, sociales y de comunicación.

\section{DISEÑO Y METODOLOGÍA DE LA INVESTIGACIÓN}

La investigación se realizó entre marzo de 2020 y marzo de 2021, tuvo un enfoque cuantitativo y correlacional para el caso de estudio. El objetivo fue diseñar y validar un modelo de competencias docentes organizado en cuatro dimensiones (pedagógica, profesional, digital y de investigación) en los AVA, incluido el soporte de laboratorios virtuales para asegurar la calidad de la educación superior en la contingencia sanitaria por la covid-19. El desarrollo del modelo de competencias docentes consideró cuatro etapas, desde su construcción hasta su validación, como se muestra en la figura 1.

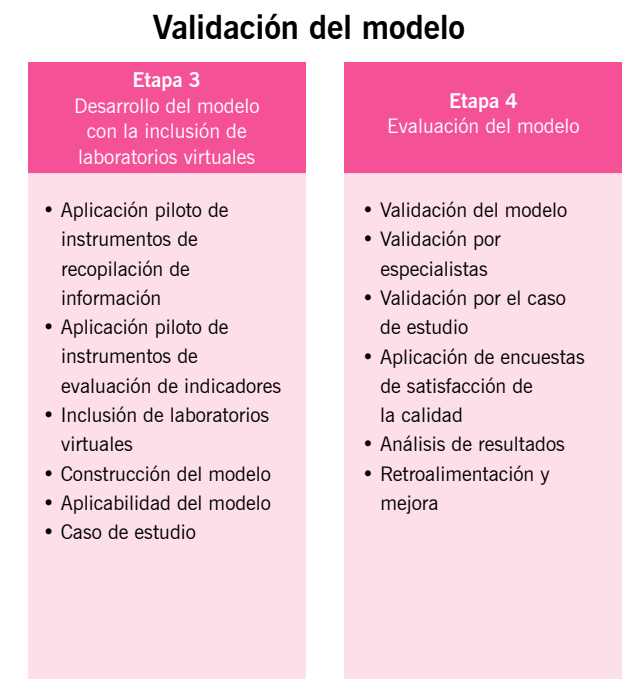

Figura 1. Esquema de construcción del modelo de competencias docentes en AVA.

Fuente: elaboración propia. 


\section{Con el fin de asegurar la} consistencia en los factores seleccionados para las dimensiones de competencias docentes, se analizaron sus indicadores y mecanismos de implementación

En la primera etapa del modelo se realizó la identificación y el diagnóstico del contexto actual. Se analizaron los referentes teóricos y se hizo la revisión de proyectos e investigaciones afines. A partir de esto, se identificaron cuatro dimensiones en las que se pueden aglutinar las diferentes competencias docentes: dimensión pedagógica, dimensión profesional, dimensión digital y dimensión de investigación. Esta es la motivación del estudio, que se enfoca en resaltar los indicadores principales dentro de cada dimensión para asegurar la calidad en los AVA.

Además, se revisaron los indicadores de aseguramiento de la calidad educativa de siete organizaciones de certificación, acreditación e instituciones de evaluación de la calidad en México y a nivel internacional, así como de los indicadores de aseguramiento de la calidad de seis modelos educativos. También se integró toda la experiencia propia, a nivel de la implementación de proyectos y cursos en AVA.

En la segunda etapa del modelo se realizó la integración de indicadores para cada dimensión definida en la etapa anterior. Esto se logró a través de la construcción de una matriz comparativa de indicadores de aseguramiento de la calidad (ver tabla 2). Se valoraron los indicadores de organismos de acreditación y de modelos educativos nacionales e internacionales relacionados con los AVA.
Como resultado del análisis comparativo en las trece instancias, se integró un tabular comparativo con los indicadores y los organismos seleccionados (los resultados se reflejan en la tabla $2 \mathrm{y}$ se desglosan en la tabla 1), en donde se muestran los indicadores de calidad utilizados por cada organismo, agrupados en cuatro dimensiones (pedagógica, profesional, digital y de investigación) y una categoría de certificación. Los indicadores de calidad dieron la pauta para identificar las competencias docentes. La experiencia, el trabajo y la retroalimentación en redes de colaboración en AVA -además de los resultados del análisis comparativo- permitieron realizar una primera validación y pilotaje del modelo a través de la retroalimentación de especialistas y tres grupos focales.

Con el fin de asegurar la consistencia en los factores seleccionados para las dimensiones de competencias docentes, se analizaron sus indicadores y mecanismos de implementación en las entidades de evaluación y acreditación nacionales e internacionales, para su incorporación dentro del tabulador de manera homologada. Se obtuvo un coeficiente de confiabilidad con un alfa de Cronbach de $91 \%$.

Los indicadores relacionados con los procesos pedagógicos de enseñanza-aprendizaje son incluidos en la mayoría de las agencias de evaluación, pero no todas utilizan indicadores para evaluar la generación y la transferencia de conocimiento. La dimensión profesional es una categoría sustantiva que permite validar el nivel de calidad en el conocimiento y la experiencia profesional en los profesores, además de su afiliación y participación en colegios y academias.

Llama la atención que los organismos de acreditación utilizan casi todos los indicadores de la dimensión digital, pero no todos evalúan la producción académica y científica de los profesores o facilitadores, ni aquellas referidas a la dimensión de investigación desarrollada en los cursos. Estos hallazgos permiten identificar áreas de oportunidad y resaltan la importancia de incluir factores 
Tabla 2. Matriz comparativa de indicadores de aseguramiento de la calidad en los organismos de acreditación, relacionados con entornos virtuales de aprendizaje

\begin{tabular}{|c|c|c|c|c|c|c|c|c|c|c|c|c|c|c|}
\hline \multirow[b]{2}{*}{ Dimensión } & \multirow[b]{2}{*}{ COMPETENCIAS DOCENTES } & \multicolumn{6}{|c|}{ Modelos EduCATIVOS } & \multicolumn{7}{|c|}{ AgENCIAS ACREDITADORAS } \\
\hline & & 崫 & 옹 & జ્ఝુ & 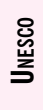 & 产 & 눙 & 㤩 & 䎹 & $\begin{array}{l}\text { 岁 } \\
\text { 岌 }\end{array}$ & 唯 & 氞 & 出 & 㗭 \\
\hline \multirow{7}{*}{ Pedagógica } & Planeación de contenidos & $\mathrm{X}$ & $\mathrm{x}$ & $\mathrm{x}$ & $\mathrm{x}$ & $\mathrm{x}$ & $\mathrm{x}$ & & $x$ & & $x$ & $x$ & & $x$ \\
\hline & $\begin{array}{l}\text { Estrategias de } \\
\text { enseñanza-aprendizaje }\end{array}$ & $\mathrm{X}$ & $\mathrm{x}$ & $\mathrm{x}$ & $x$ & $x$ & $\mathrm{x}$ & $x$ & $\mathrm{x}$ & $\mathrm{x}$ & $\mathrm{X}$ & $x$ & $x$ & $x$ \\
\hline & Evaluación del aprendizaje & $\mathrm{X}$ & $\mathrm{x}$ & $x$ & $\mathrm{x}$ & $x$ & $\mathrm{x}$ & $x$ & $\mathrm{x}$ & $x$ & $x$ & $x$ & $x$ & $\mathrm{x}$ \\
\hline & Comunicación efectiva & $x$ & $x$ & $x$ & $x$ & $x$ & $x$ & $x$ & $x$ & & & $x$ & & $x$ \\
\hline & Liderazgo & $\mathrm{X}$ & & $\mathrm{x}$ & $\mathrm{x}$ & & $\mathrm{x}$ & & $\mathrm{x}$ & & $x$ & & & \\
\hline & $\begin{array}{l}\text { Generación y transferencia } \\
\text { del conocimiento }\end{array}$ & $\mathrm{X}$ & $x$ & & & $x$ & & $x$ & & $x$ & & $\mathrm{x}$ & & \\
\hline & Instrucción personalizada & $\mathrm{X}$ & $x$ & $\mathrm{X}$ & $\mathrm{x}$ & & $\mathrm{x}$ & $x$ & & $\mathrm{X}$ & $x$ & $\mathrm{x}$ & $x$ & $x$ \\
\hline \multirow{3}{*}{ Profesional } & $\begin{array}{l}\text { Trayectoria en } \\
\text { entrenamiento docente }\end{array}$ & $\mathrm{X}$ & $\mathrm{x}$ & $x$ & & $x$ & $x$ & $x$ & $x$ & $x$ & $x$ & $x$ & $x$ & $x$ \\
\hline & Experiencia profesional & $\mathrm{X}$ & $x$ & $x$ & $x$ & $x$ & $x$ & $x$ & $x$ & $x$ & $x$ & & & $x$ \\
\hline & $\begin{array}{l}\text { Pertenencia a colegios y } \\
\text { academias }\end{array}$ & & $x$ & $x$ & $\mathrm{x}$ & & $\mathrm{x}$ & $x$ & $\mathrm{x}$ & & & $\mathrm{x}$ & $x$ & $\mathrm{x}$ \\
\hline \multirow{3}{*}{ Digital } & $\begin{array}{l}\text { Entrenamiento en el uso } \\
\text { de TIC y herramientas } \\
\text { digitales }\end{array}$ & $\mathrm{X}$ & $\mathrm{x}$ & $x$ & $\mathrm{x}$ & $\mathrm{x}$ & $\mathrm{X}$ & $\mathrm{X}$ & & $\mathrm{X}$ & $x$ & $\mathrm{x}$ & $\mathrm{X}$ & $\mathrm{x}$ \\
\hline & $\begin{array}{l}\text { Experiencia en cursos } \\
\text { de educación a distancia } \\
\text { utilizando medios digitales }\end{array}$ & $x$ & $\mathrm{x}$ & $x$ & $x$ & $x$ & $x$ & $x$ & $x$ & $x$ & $x$ & $x$ & $x$ & $x$ \\
\hline & $\begin{array}{l}\text { Experiencia en ambientes } \\
\text { multimodales e híbridos }\end{array}$ & $x$ & $x$ & $x$ & $x$ & & $\mathrm{x}$ & & & & $\mathrm{x}$ & $x$ & & $x$ \\
\hline \multirow{2}{*}{ Investigación } & Producción académica & & $x$ & $x$ & & & & $x$ & $x$ & $\mathrm{x}$ & & $x$ & $x$ & \\
\hline & Producción científica & & $x$ & $x$ & & & & & $x$ & $\mathrm{x}$ & & $x$ & $x$ & \\
\hline \multirow{4}{*}{ Certificación } & $\begin{array}{l}\text { Competencias de } \\
\text { enseñanza }\end{array}$ & $x$ & & & & $x$ & $x$ & $x$ & & & & & & \\
\hline & Competencias digitales & $x$ & & & $\mathrm{x}$ & $x$ & $\mathrm{x}$ & $x$ & & & & & & \\
\hline & $\begin{array}{l}\text { Competencias } \\
\text { profesionales }\end{array}$ & $x$ & & & & & & $x$ & & & & $x$ & & \\
\hline & $\begin{array}{l}\text { Competencias de } \\
\text { investigación }\end{array}$ & & & & & & & & & $\mathrm{X}$ & & $x$ & & \\
\hline
\end{tabular}

Fuente: elaboración propia. 
relacionados con la certificación de competencias en las cuatro dimensiones identificadas como parte de los indicadores de calidad.

En esta fase de análisis de confiabilidad se desarrolló y aplicó una encuesta de satisfacción a 100 estudiantes, divididos en doce grupos, de cinco programas académicos universitarios para la validación de la integración correcta de las dimensiones. Los programas académicos considerados fueron de las disciplinas educativas, tecnológicas, biológicas, negocios y sociales. Asimismo, se diseñó un instrumento de seguimiento de la calidad educativa para realizar un acompañamiento por profesores investigadores que denominamos enlaces de seguimiento.

En la tercera etapa se construyó el modelo de competencias docentes para ambientes virtuales de aprendizaje. Se sintetizaron los resultados de la información obtenida en las etapas previas, la revisión de referentes teóricos y los resultados de un análisis comparativo de indicadores de calidad académica para AVA, así como el análisis de información obtenida a través de los instrumentos diseñados y aplicados en la etapa anterior.

La construcción del modelo permitió integrar las competencias docentes en cada dimensión.
De la misma forma, se estableció el requerimiento de las certificaciones docentes como otro elemento de aseguramiento de la calidad educativa. Para la implementación del caso de estudio se consideró la totalidad de la población estudiantil (9 980 alumnos), que conforma el nivel de educación superior en todas las modalidades y programas académicos de la Universidad Popular Autónoma del Estado de Puebla (UPAEP), durante los períodos de primavera, verano y otoño de 2020, así como primavera de 2021.

La implementación permitió valorar la incorporación de las competencias y los indicadores del modelo en los AVA, que incluía la articulación de laboratorios virtuales para el fortalecimiento en la formación, con estándares de calidad educativa. Esta etapa finalizó con una retroalimentación de grupos focales y de especialistas para el enriquecimiento del modelo y su presentación final.

El modelo completo de competencias docentes en AVA se muestra en la figura 2. En la parte central de este se encuentra el entorno de mediación pedagógica, enfocada en el aprendizaje de los estudiantes, apoyado en el ámbito académico, las áreas administrativas y de servicios, y respaldado por la calidad educativa. En este modelo, el estudiante no

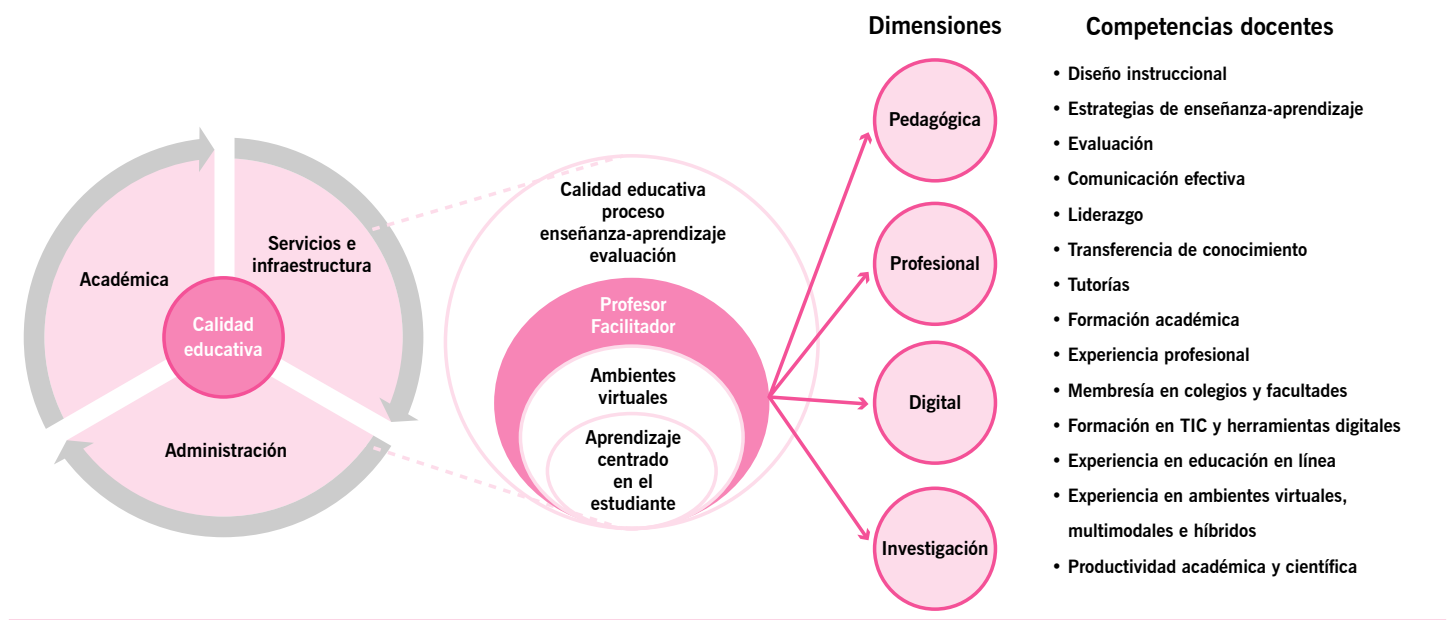

Figura 2. Modelo de competencias docentes para AVA.

Fuente: elaboración propia. 
solo tiene un rol activo, sino que enfatiza la participación activa del docente, quien requiere de diversas competencias para desempeñarse en los AVA.

Estas competencias permiten conformar un perfil docente integral, con la definición de indicadores claros que aseguren la calidad educativa en los AVA (ver tabla 3). Además, estos elementos permiten la elaboración de programas de capacitación, evaluación, seguimiento y mejora de la calidad en la educación superior.
En la cuarta etapa se realizó la validación del modelo, que incluye la retroalimentación por especialistas, los resultados del caso de estudio obtenidos de una encuesta de satisfacción de la calidad (ver figura 3) aplicada en los cuatro períodos académicos comprendidos entre marzo de 2020 y marzo de 2021, con una participación de más de $20 \%$ de la población estudiantil, así como un proceso de seguimiento y evaluación de la calidad educativa con 41 profesores investigadores como enlaces de calidad.

Tabla 3. Indicadores de competencias docentes para el aseguramiento de la calidad educativa en los AVA

\begin{tabular}{|c|c|c|}
\hline Dimensión & COMPETENCIA DOCENTE / INDICADOR DE EVALUACIÓN & Certificaciones \\
\hline \multirow{7}{*}{ Pedagógica } & Diseño instruccional & \multirow{7}{*}{ Competencias pedagógicas certificadas } \\
\hline & Estrategias de enseñanza-aprendizaje & \\
\hline & Evaluación y retroalimentación & \\
\hline & Comunicación efectiva & \\
\hline & Liderazgo & \\
\hline & Transferencia del conocimiento & \\
\hline & Tutorías & \\
\hline \multirow{3}{*}{ Profesional } & Formación académica & \multirow{3}{*}{ Competencias profesionales certificadas } \\
\hline & Experiencia profesional & \\
\hline & Membresías en colegios y academias & \\
\hline \multirow{3}{*}{ Digital } & Formación en TIC y herramientas digitales & \multirow{3}{*}{ Competencias digitales certificadas } \\
\hline & Experiencia en ambientes virtuales de aprendizaje & \\
\hline & Experiencia con laboratorios virtuales & \\
\hline \multirow{2}{*}{ Investigación } & Productividad académica & \multirow{2}{*}{ Competencias de investigación } \\
\hline & Productividad científica & \\
\hline
\end{tabular}

Fuente: elaboración propia.

¿Consideras que se lograron cumplir los aprendizajes esperados en la asignatura?

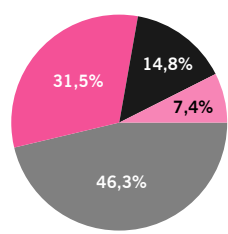

Totalmente de acuerdo

De acuerdo

Ni de acuerdo ni en desacuerdo

- En desacuerdo

Totalmente en desacuerdo

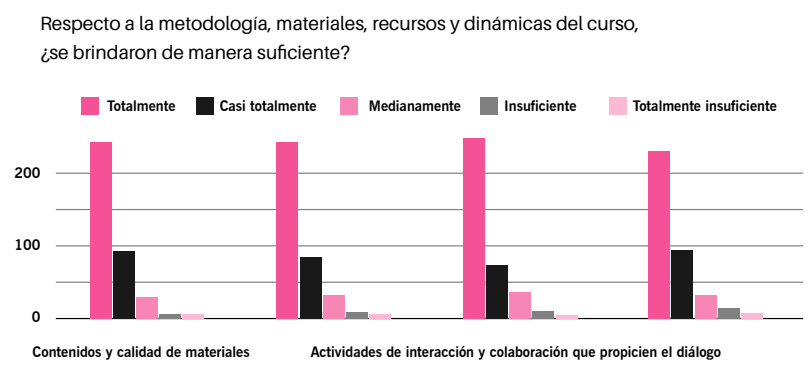

Respecto a la metodologia, materiales, recursos y dinámicas del curso, ¿se brindaron de manera suficiente?

Figura 3. Ejemplos de los resultados de la encuesta de satisfacción de la calidad. Fuente: elaboración propia. 
Los principales pasos para la validación del modelo se describen a continuación.

\section{a) Contexto educativo: ambientes virtuales de aprendizaje}

En congruencia con la elaboración del modelo, el contexto en el que se llevó a cabo su validación fue la implementación de la totalidad de los cursos en AVA en la UPAEP, a raíz de la pandemia por la covid-19. Así, se definieron lineamientos de aseguramiento del aprendizaje para las asignaturas prácticas y de laboratorio, durante las cuales se utilizaron plataformas educativas, sesiones de video, conferencias síncronas y simuladores especializados (laboratorios virtuales). Estos últimos empleados principalmente para las áreas de negocios, ciencias biológicas, ingenierías y ciencias médicas. Adicionalmente, su operación se aseguró a través del diseño de videograbaciones de las actividades prácticas formativas de cada asignatura.

La figura 4 muestra una de las actividades de simulación con software especializado para los procesos productivos.

\section{b) Participantes}

Se aplicaron encuestas de satisfacción a todos los grupos, en cada uno de los períodos académicos señalados, con una participación total de 5650 estudiantes.

\section{c) Variables e instrumento}

El primer instrumento aplicado fue una encuesta de satisfacción a estudiantes, con un cuestionario tipo Likert, que tuvo como propósito principal validar las cuatro dimensiones del modelo como variables centrales del estudio. El instrumento constó de 19 ítems, distribuidos de la siguiente forma: seis en la dimensión pedagógica, seis en la dimensión digital, cuatro en la dimensión profesional y tres para la dimensión de investigación. El segundo instrumento de validación de implementación del modelo de calidad educativa en AVA fue el formulario de seguimiento de la cátedra por aula virtual, el cual incluyó más de 700 grupos observados en promedio, por período académico.

\section{RESULTADOS}

La aplicación del instrumento de validación que evaluó las dimensiones pedagógica, profesional, digital y de investigación, arrojó los siguientes resultados a partir de los indicadores de cada dimensión. Como se muestra en la gráfica 1, la integración de los indicadores de calidad de las prácticas docentes en términos de planeación de contenidos, comunicación efectiva, tutoría y evaluación del aprendizaje, requiere considerar las habilidades y las competencias integrales

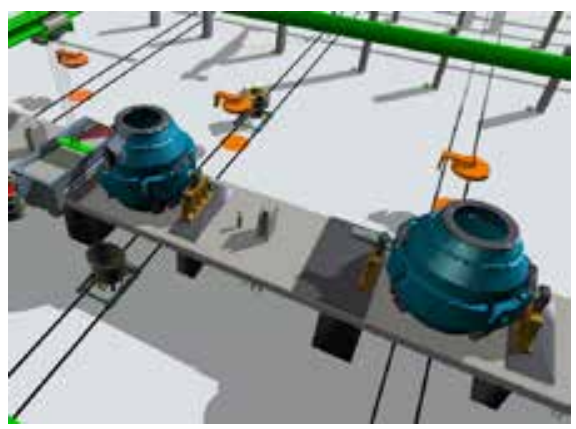

Figura 4. Laboratorio de simulación de manufactura.

Fuente: imagen de práctica de laboratorio del proceso de manufactura en la plataforma SolidWorks. 
docentes, de manera que se enfatice el liderazgo en el aula, así como la generación y transferencia de conocimiento.

En la gráfica 2 se pueden observar algunos puntajes menores en las categorías de planeación y organización de los contenidos de las asignaturas en la plataforma tecnológica, la gestión y explotación de las herramientas digitales, la in- corporación de estrategias de enseñanza-aprendizaje en AVA y la evaluación del aprendizaje a través de los medios digitales. Los factores de alta puntuación destacables son el diseño instruccional, así como la generación y transferencia de conocimiento, lo que explica la percepción favorable de integrar estos aspectos en el aula virtual.

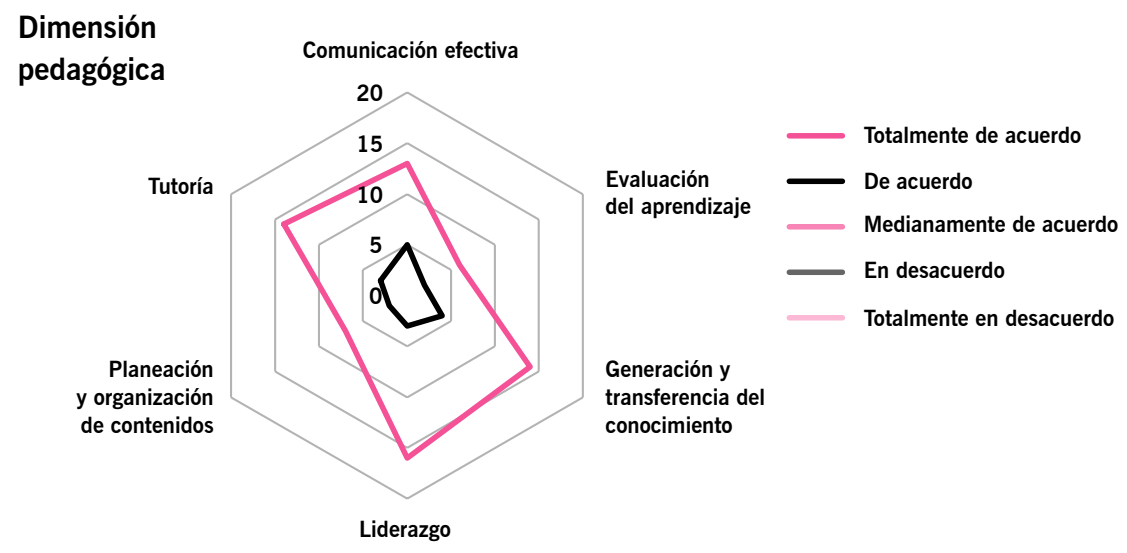

Gráfica 1. Resultados de la encuesta de satisfacción a estudiantes universitarios sobre la dimensión pedagógica en entornos apoyados por tecnología. Fuente: elaboración propia.

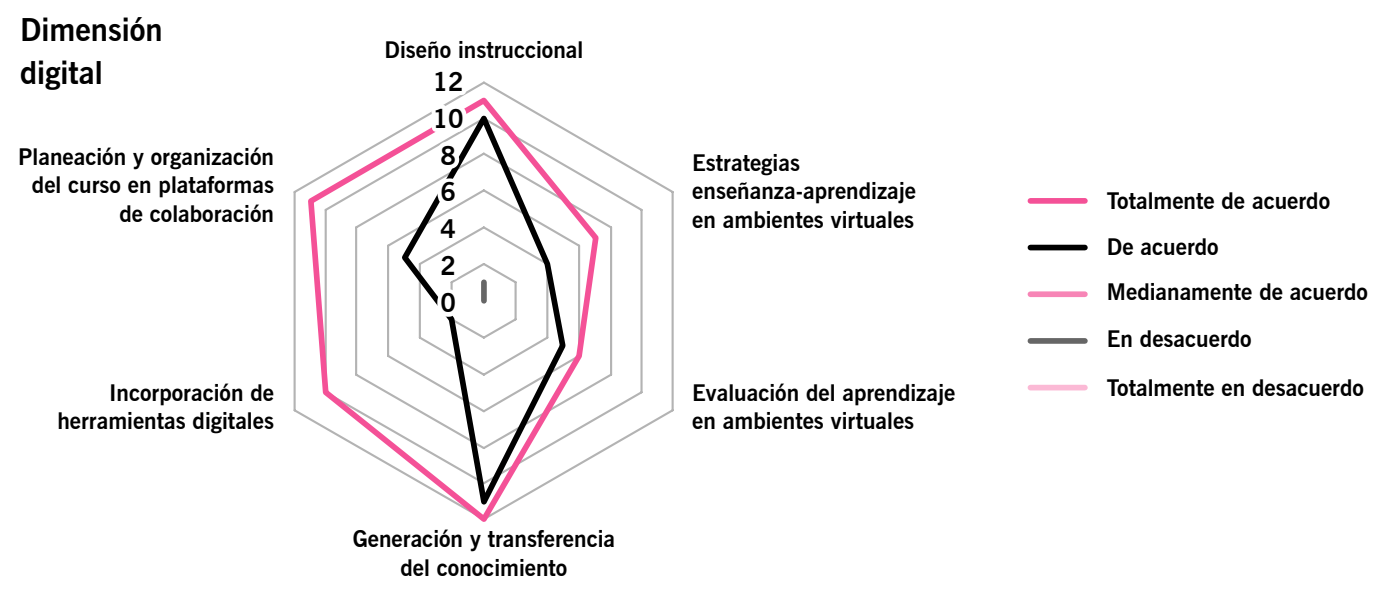

Gráfica 2. Resultados de la encuesta de satisfacción a estudiantes sobre la dimensión digital en entornos apoyados por tecnología. Fuente: elaboración propia. 
En la gráfica 3 se puede apreciar que las certificaciones y la experiencia profesional son los criterios altamente valorados por los estudiantes, seguidos de la adscripción de membresías a colegios y la formación académica, lo cual implica una buena integración de los indicadores para evaluar la dimensión profesional en los docentes.

La gráfica 4 muestra una relación congruente entre la productividad académica y la científica, principalmente. También se identifica la necesidad de reforzar la formación de los docentes para fortalecer esta dimensión de investigación.

\section{DISCUSIÓN}

Diferentes estudios sobre la calidad educativa concuerdan en que no existen dimensiones de calidad de la educación superior aceptadas uni- versalmente. $\mathrm{Al}$ respecto, se han propuesto numerosas dimensiones que son difíciles de evaluar en un solo estudio; además de que existe una incapacidad para captar las opiniones de diferentes partes interesadas (profesores, personal administrativo, jefes, estudiantes, entre otras) (Kumar et al., 2020; Prakash, 2018; Kundu, 2017). Esto parece sugerir la necesidad de que las IES desarrollen sus propios modelos, de manera sistemática y rigurosa, a partir de las necesidades específicas de evaluación de la calidad, al mismo tiempo que se contemplan las exigencias de calidad internacionales.

Los resultados de la validación del presente estudio permiten afirmar que las dimensiones seleccionadas para integrarse en el modelo son pertinentes al contexto actual, así como adecuadas para señalar las características de calidad que requieren los AVA, de acuerdo con criterios

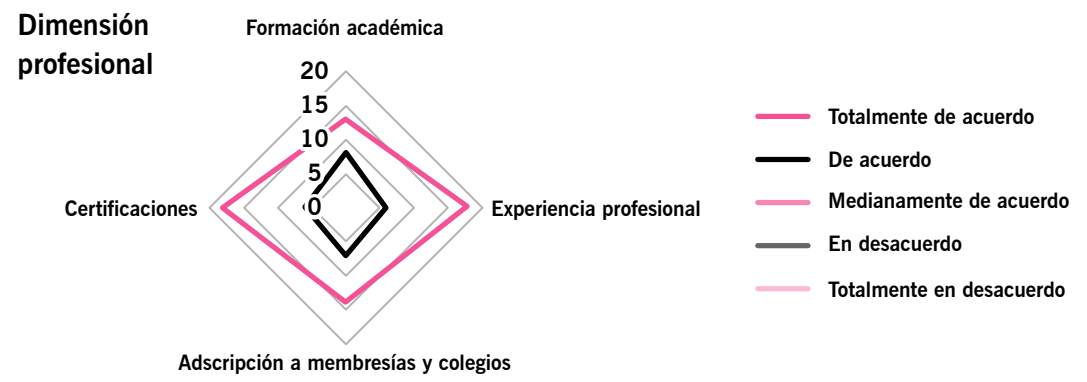

Gráfica 3. Resultados de la encuesta de satisfacción de la calidad a estudiantes sobre la dimensión profesional en entornos apoyados por tecnología. Fuente: elaboración propia.

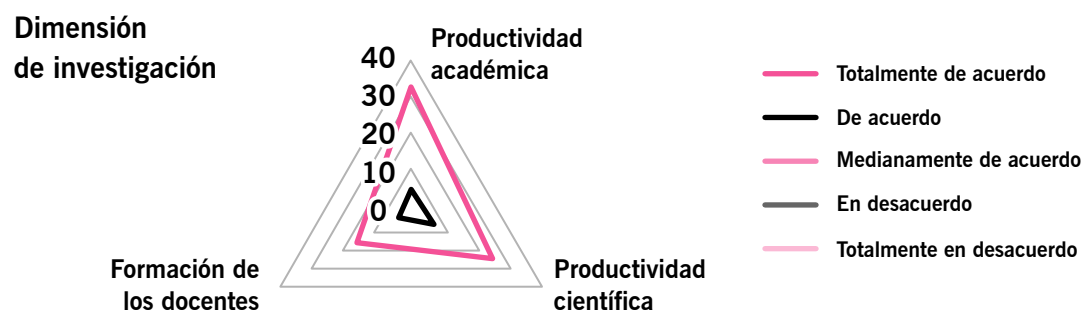

Gráfica 4. Resultados de la encuesta de satisfacción a estudiantes sobre la dimensión de investigación en entornos apoyados por tecnología. Fuente: elaboración propia. 
e indicadores de organismos certificadores nacionales e internacionales y los modelos educativos analizados. Adicionalmente, se identificaron fortalezas y debilidades institucionales, respecto a sus propios indicadores de calidad durante la contingencia de salud por la covid-19.

En cuanto a la dimensión pedagógica, nuestros hallazgos coinciden con estudios como el de Zempoalteca et al. (2016), en que es necesario reforzar las habilidades docentes, como la de diseño instruccional y evaluación de aprendizajes, explicable debido a que los profesores de las IES carecen de formación docente especializada y certificada en su mayoría.

Asimismo, en cuanto a la dimensión digital se coincide con otros estudios (Scherer et al., 2020) en que se requiere más acompañamiento y capacitación a los docentes respecto a la planeación de contenidos, el uso de herramientas de enseñanza-aprendizaje y de evaluación en ambientes digitales. También se detectó que se requiere incorporar una capacitación avanzada en competencias digitales a la totalidad de la planta docente, además de implementar un seguimiento y evaluación continua de la calidad a lo largo de cada período académico.

En cuanto a la dimensión profesional, se identificó la necesidad de fortalecer las competencias profesionales con la certificación y el reconocimiento de la experiencia práctica en los sectores. Respecto a la dimensión de investigación se detectó la necesidad de generación de estrategias que propicien el desarrollo de proyectos de investigación con fuertes estándares metodológicos.

Una fortaleza del modelo propuesto es que su diseño se basa en los estándares de calidad educativa nacionales e internacionales, con lo que se intenta responder a los cambios educativos forzados que implicó la crisis sanitaria internacional. Además, representa una innovación y llena un vacío respecto a estudios de calidad educativa basados en la percepción de los estudiantes, así como de modelos para evaluar la calidad docente en los AVA.
Como parte de las debilidades del modelo que se identificaron, se encuentra que se requiere desarrollar la ponderación de indicadores y categorías. Esto podría realizarse a través de la replicación de la implementación del modelo en otras instituciones educativas, mediante el uso de AVA. También es factible integrar de forma más robusta la opinión de los propios docentes en la mejora del modelo.

\section{CONCLUSIONES}

Este estudio aporta un modelo de competencias docentes en AVA para asegurar la calidad de la educación superior durante la contingencia de salud por la covid-19. La validación del modelo indica su pertinencia para evaluar la calidad educativa, a partir de la percepción de los estudiantes. Los resultados de la validación permitieron constatar la pertinencia del modelo en cada una de sus dimensiones, así como identificar las fortalezas y las debilidades en las competencias de los docentes.

Este modelo podría ser la base para propuestas y estudios similares que ensayen estas y otras dimensiones para evaluar la calidad educativa de los AVA en las instituciones de educación superior. Se requiere avanzar en la indagación de estrategias pertinentes de acompañamiento a los docentes para superar sus brechas en uso educativo de las TIC y otras herramientas digitales. a

\section{REFERENCIAS}

Aguilar, N. J. (2020). Continuidad pedagógica en el nivel medio superior: acciones y reacciones ante la emergencia sanitaria. En IISUE, Educación y pandemia. Una visión académica (pp. 4754). México: UNAM. https://www.iisue.unam.mx/investigacion/ textos/educacion_pandemia.pdf

Albrahim, F. A. (2020). Online teaching skills and competencies. Turkish Online Journal of Educational Technology-TOJET, 19(1), pp. 9-20. https://eric.ed.gov/?q= source\%3A\%22Turkish+ Online + Journal + of + Educational + Technology $+\% 22 \& i d=$ EJ1239983 
Allam, Z. (2018). Students' perception of quality in higher education: An empirical investigation. Management Science Letters, 8(5), pp. 437-444. https://doi.org/10.5267/j.msl.2018.4.002

Ally, M. (2019). Competency profile of the digital and online teacher in future education. International Review of Research in Open and Distributed Learning, 20(2). https://doi.org/10.19173/irrodl.v20i2.4206

Barrón Tirado, M. C. (2020). La educación en línea. Transiciones y disrupciones. En IISUE, Educación y pandemia. Una visión académica (pp. 66-74). México: UNAM. http://www.iisue. unam.iisue/covid/educacion-y-pandemia

Bonilla Trujillo, D.; Villamil Reyes, V. V. y Mora Montes, J. F. (2019). Uso de simuladores 3D como estrategia tecnopedagógica para la transferencia de conocimiento en el aprendizaje de la anatomía animal. Documentos de Trabajo ECAPMA, (1). https://doi. org/10.22490/ECAPMA.3414

Cabero-Almenara, J. y Llorente-Cejudo, C. (2020). Covid-19: transformación radical de la digitalización en las instituciones universitarias. Campus Virtuales, 9(2), pp. 25-34. http:// www.uajournals.com/ojs/index.php/campusvirtuales/article/ view/713

Clavijo, D. (2018). Competencia del docente universitario en el siglo XXI. Revista Espacios, 39(20). https://www.revistaespacios. com/a18v39n20/a18v39n20p22.pdf

Cocunubo-Suárez, J. I.; Parra-Valencia, J. A. y Otálora-Luna, J. E. (2018). Propuesta para la evaluación de entornos virtuales de enseñanza aprendizaje con base en estándares de usabilidad. TecnoLógicas, 21(41), pp. 135-147. https://doi. org/10.22430/22565337.732

El Alfy, S. \& Abukari, A. (2020). Revisiting perceived service quality in higher education: Uncovering service quality dimensions for postgraduate students. Journal of Marketing for Higher Education, 30(1), pp. 1-25. https://doi.org/10.1080/08841241.2019 .1648360

García-Chitiva, M. D. P. y Suárez-Guerrero, C. (2019). Estado de la investigación sobre la colaboración en entornos virtuales de aprendizaje. Píxel-Bit: Revista de Medios y Educación, (56), pp. 169-191. https://doi.org/10.12795/pixelbit.2019.i56.09

García-Peñalvo, F. J.; Corell, A.; Abella-García, V. y Grande, M. (2020). La evaluación online en la educación superior en tiempos de la covid-19. Education in the Knowledge Society, 21. https://doi.org/10.14201/eks.23013

González Hernández, N. Y. y Victoria Uribe, R. (2019). Nuevas alternativas para transferencia de conocimiento sustentable: el uso de interfaces de realidad virtual. En J. A. Vargas Castro y G. A. García Luna Villagrán (comps.), Diseño Estratégico e Incluyente para un Desarrollo Sostenible. México: Universidad Autónoma del Estado de México. http://coloquio2019.faduaemex.org.mx/wp-content/uploads/2019/11/design-estrategicweb.pdf\#page $=10$

Herrera, L.; Souza, M. R. y de Quadros, J. F. (2018). Evaluación de la calidad en la educación superior: una revisión de la literatura a partir de la satisfacción del alumnado. Cadernos de pesquisa, 25(2), pp. 71-89. http://dx.doi.org/10.18764/2178-2229. v25n2p71-89

Jiménez Moreno, J. A. (2019). La evaluación y acreditación de la educación profesional en México: ila legitimación y competitividad como fin de la universidad? Revista de la educación superior, 48(189), pp. 55-72. https://doi.org/10.36857/ resu.2019.189.614

Khlaisang, J. \& Songkram, N. (2019). Designing a virtual learning environment system for teaching twenty-first century skills to higher education students in ASEAN. Technology, Knowledge and Learning, 24(1), pp. 41-63. http://dx.doi.org/10.1007/ s10758-017-9310-7

Kumar, P.; Shukla, B. \& Passey, D. (2020). Impact of Accreditation on Quality and Excellence of Higher Education Institutions. Investigación Operacional, 41(2), pp. 151-167. https://eprints.lancs. ac.uk/id/eprint/141916

Kundu, G. K. (2017). Quality in higher education from different perspectives: a literature review. International journal for quality research, 11(1), pp. 17-35. http://dx.doi.org/10.18421/ IJQR11.01-02

Luna Encalada, W.; Costales, P. M.; Cordovez Machado, S. P. \& Guaiña Yungán, J. (2020). Virtual Laboratories in Virtual Learning Environments. En The International Conference on Advances in Emerging Trends and Technologies (pp. 114-126). Springer, Cham. https://doi.org/10.1007/978-3-030-63665-4_9

Macías-Catagua, 0. W. (2018). El desarrollo de competencias genéricas en el docente universitario. Dominio de las Ciencias, 4(3), pp. 240-252. http://dx.doi.org/10.23857/dc.v4i3.806

Nechypurenko, P. P.; Selivanova, T. V. \& Chernova, M. P. (2019). Using the cloud-oriented virtual chemical laboratory lab in teaching the solution of experimental problems in chemistry of 9 th grade students. En Proceedings of the 15th International Conference on ICT in Education, Research and Industrial Applications. Integration, Harmonization and Knowledge Transfer (pp. 968983). CEUR Workshop Proceedings. 
Peterson-Ahmad, M. B.; Pemberton, J. \& Hovey, K. A. (2018). Virtual learning environments for teacher preparation. Kappa Delta $\mathrm{Pi}$ Record, 54(4), pp. 165-169. https://doi.org/10.1080/0022895 8.2018.1515544

Phungsuk, R.; Viriyavejakul, C. \& Ratanaolarn, T. (2017). Development of a problem-based learning model via a virtual learning environment. Kasetsart Journal of Social Sciences, 38(3), pp. 297-306. https://doi.org/10.1016/.kjss.2017.01.001

Prakash, G. (2018). Quality in higher education institutions: insights from the literature, The TQM Journal, 30(6), pp. 732-748. https://doi.org/10.1108/TQM-04-2017-0043

Salmerón-Manzano, E. \& Manzano-Agugliaro, F. (2018). The Higher Education Sustainability through Virtual Laboratories: The Spanish University as Case of Study. Sustainability, 10(11), p. 4040. https://doi.org/10.3390/su10114040

Sánchez Cuevas, M. (2020). Chapter 19. Challenge Based Learning (CBL). En Education Applications \& Developments V. Lisboa: inScience Press.

Sari, T. \& Nayir, F. (2020). Challenges in Distance Education During the (Covid-19) Pandemic Period. Qualitative Research in Education (2014-6418), 9(3), pp. 328-360. http://dx.doi. org/10.17583/qre.2020.5872
Scherer, R.; Howard, S. K.; Tondeur, J. \& Siddiq, F. (2020). Profiling teachers' readiness for online teaching and learning in higher education: Who's ready? Computers in Human Behavior, 118, 106675. https://doi.org/10.1016/..chb.2020.106675

Tobón, S. (2008). Gestión curricular y ciclos propedéuticos. Bogotá: ECOE.

Villarroel, V. A. y Bruna, D. V. (2017). Competencias pedagógicas que caracterizan a un docente universitario de excelencia: un estudio de caso que incorpora la perspectiva de docentes y estudiantes. Formación universitaria, 10(4), pp. 75-96. http:// dx.doi.org/10.4067/S0718-50062017000400008

Yong-Sik, H. \& Yung Kyun, C. (2019). Higher education service quality and student satisfaction, institutional image, and behavioral intention. Social Behavior \& Personality: An International Journal, 47(2), pp. 1-12. https://doi.org/10.2224/ sbp.7622

Zempoalteca Durán, B.; Barragán López, J. F.; González Martínez, J. y Guzmán Flores, T. (2016). Formación en TIC y competencia digital en la docencia de instituciones públicas de educación superior. Apertura, 9(1), pp. 80-96. http://dx.doi.org/10.32870/ Ap.v9n1.922

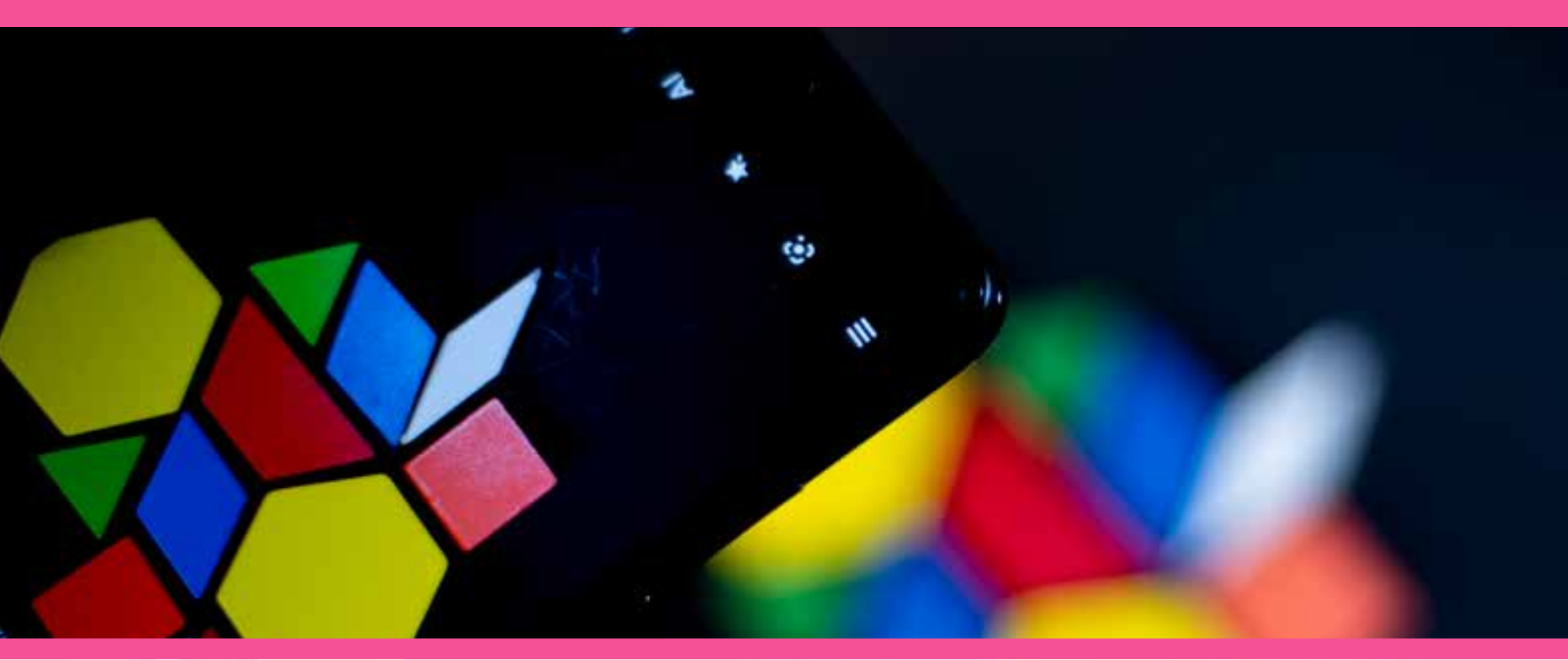

Este artículo es de acceso abierto. Los usuarios pueden leer, descargar, distribuir, imprimir y enlazar al texto completo, siempre y cuando sea sin fines de lucro y se cite la fuente.

\section{CÓMO CITAR ESTE ARTíCULO:}

Vega Lebrún, Carlos Arturo; Sánchez Cuevas, Mariano; Rosano Ortega, Genoveva y Amador Pérez, Silvia Elena. (2021). Competencias docentes, una innovación en ambientes virtuales de aprendizaje en educación superior. Apertura, 13(2), pp. 6-21. http://dx.doi.org/10.32870/ Ap.v13n2.2061 